Research Article

\title{
Theoretical Analysis of Heat Stress Prefabricating the Crack in Precision Cropping
}

\author{
Lijun Zhang* and Yongrui Zhao
}

\author{
College of Electromechanical Engineering, China University of Petroleum, Qingdao, 266580-China
}

Received 15 May 2013; Accepted 25 July 2013

\begin{abstract}
The mathematical model of the metal bar in course of heat treatment is built by regarding the convective heat transfer process of the metal bar as the heat conduction boundary condition. By the theory analysis and numerical simulation methods, the theoretical expression of unsteady multidimensional temperature field for the axisymmetric model of metal bar is obtained. Temperature field distribution of bar V-shaped notch equivalent tip is given by ANSYS software. The quantitative relationship between temperature of bar inner key points and the time is determined. Through the polynomial curve fitting, the relation between the ultimate strength and the temperature is also given. Based on it, the influences of the width of the adiabatic boundary and water velocity on the critical temperature gradient of germinating heat crack in the tip of V-shaped notch are analyzed. The experimental results in precision cropping show that the expression of unsteady multidimensional temperature field is feasible in the rapid calculation of crack generation.
\end{abstract}

Keywords: Precision cropping, Heat stress, Crack, V-shaped notch, Temperature field

\section{Introduction}

The precision cropping of metal bar is widely used in industrial production, such as chain pins, micro-motor shafts and combustion engineer appurtenances and so on. High speed shearing cropping, radial holding differential cropping, and blanking under the high axis load are effective and economical methods of bar cutting [1]. The shear behaviours of bar fine-blanking and cropping processes through their experimental performance have been studied. In order to investigate the phenomena of shearing behaviours and effects during the cutting operation, some major parameters, such as the surface finish, hardness distribution are also examined by employing the certain equipment [2]. Unlike these common shear methods, where a cropping die is used so as to cause the huge shearing force and the defective fracture surfaces, a low-stress cropping method is proposed where the gap effect and bend effect of annular V-shaped notch of bar surface are considered as factors to improve breakability and surface finish and to decrease cutting force and tool wear [3, 4]. In the cropping process, $\mathrm{V}$-shaped notches are cut at specific length intervals in metal bar to engender the stress concentration at the bottom of the V-shaped notch. Then the slotted bar is fed to crop under the action of continuous bending fatigue force and would rupture after certain periods. It is obvious that the stress concentration degree of V-shaped notch bottom has a direct relation to the surface quality of rupture section and the cropping time. However, by large numbers of the lowstress cropping experiments, it is found that the cropping cross-section quality can be kept high, but the cropping time

*E-mail address: zli-2@163.com

ISSN: 1791-2377 @ 2013 Kavala Institute of Technology. All rights reserved. is still longer because the radius at the V-shaped notch bottom can not be sufficiently small, where the V-shaped notch is mainly obtained by general turning method $[5,6]$. On the basis of low-stress cropping, the method of prefabricating the fine crack at V-shaped notch bottom by heat stress was proposed $[7,8,9,10]$. However, the axisymmetric model with unsteady multidimensional temperature field for different bar material is still not built theoretically, and the critical temperature gradient in the bar is not also presented, which results in complexity of calculation course of germinating heat crack. Therefore, by the theory analysis and the numerical simulation methods, the theoretical expression of unsteady multidimensional temperature field for the axisymmetric model is obtained. Temperature field distribution of equivalent tip point of $\mathrm{V}$ shaped notch in the bar is determined. In addition, the influences of the width of the adiabatic boundary and water velocity on the critical temperature gradient are also analyzed in detail.

\section{Mechanism of High-speed Circumferential Low-stress Bending Fracture Cropping}

The mechanical parts of the cropping system with highspeed circumferential low-stress bending fracture mainly consist of the transmission system, the blanking die, the fine tuning system, the hydraulic clamping and the feeding mechanism, and the body. The working mechanism of blanking die and fine tuning system is shown in Fig.1. When the cropping machine works, the variable frequency motor drives the column hammer mounted on the eccentric spindle by the two-stage accelerative transmission system. The 
column hammer made by antifriction composite provides the bar with circumferentially continuous eccentric loading. The ball screw controlled by the AC servo motor drives the feed sleeve to adjust the compressive deformation of the column hammer to make the crack of V-shaped notch tip extend flexibly and regularly. In course of cropping, the clamping die can confine the radial movement, the radial torsion and the axial torsion of metal bar. The feed-in device, by which the arm of the exciting force can be adjusted, can restrict the axial movement of metal bar. On this basis, the low-stress bend precise cropping can be carried out.
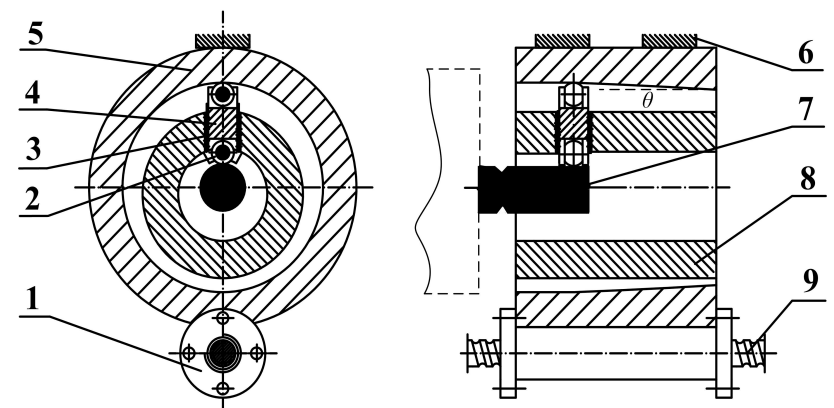

Fig. 1. Working mechanisms of blanking die and fine tuning system 1. Nut and accessory 2. Roller 3. Ball sleeve 4. Column hammer 5. Feed sleeve 6. Sliding guide 7. Bar 8. Eccentric spindle 9. Ball screw

\section{Theoretical analysis of heat stress prefabricating the fine crack}

3.1 Fundamental of prefabricating the fine crack at the V-shaped notch bottom

The basic principle of heat stress prefabricating the fine crack at the V-shaped notch bottom is mainly based on heat expansion and cold retraction effect of metal material. As a result of temperature gradient along the axial different parts of the metal bar, the heat tension stress occurs in the inner of the slotted bar. The heat tension stress in the vicinity of Vshaped notch tip is maximal because of stress concentration effect. The fine crack in the vicinity of V-shaped notch tip will appear when the heat tension stress reaches the ultimate strength of the metal material [11].

According to above principle, the cooling mode of the metal bar in heat treatment is shown in Fig.2. The bar with annular slots is heated to the settled temperature firstly, about $500^{\circ} \mathrm{C}$, and the $\mathrm{V}$-shaped parts of the bar are protected with asbestos clapboards and then the bar is cooled rapidly by cooling water. In the course of cooling, the temperature gradient between the inner and surface of the bar and that between $\mathrm{V}$-shaped notch part and the bar surface acted on the cooling water will appear. By setting reasonably parameters, the fine crack will occur in the vicinity of $\mathrm{V}$ shaped notch tip firstly due to the action of threedimensional stress of the V-shaped notch.

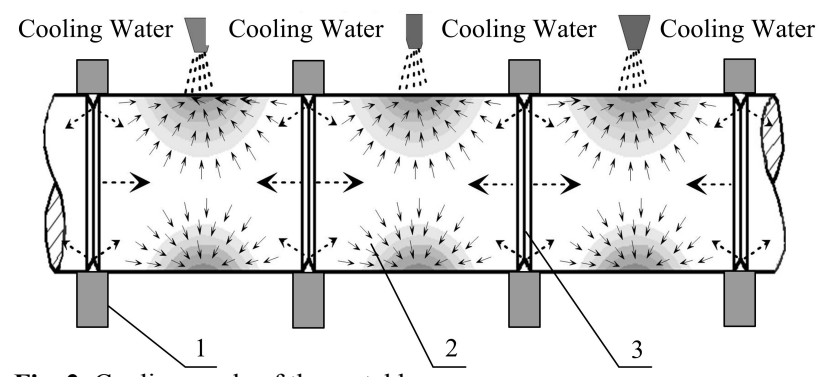

Fig. 2. Cooling mode of the metal bar

1. Asbestos clapboard 2. Slotted bar 3. V-shaped notch

\subsection{Axisymmetric model with unsteady multidimensional temperature field}

To build the differential equation of the heat conduction of bar inner points, and give the relation between the temperature gradient and the time of the corresponding point in the bar, the mathematical model of the metal bar in course of heat treatment is shown in Fig.3. As is shown in Fig.3, point $\mathrm{A}$ is the boundary point in the bar and it is also the midpoint of bar surface acted by the cooling water. The radius of point $\mathrm{B}$ is equal to the radius of $\mathrm{V}$-shaped notch tip point $\mathrm{D}$. Point $\mathrm{E}$ is a point in the condition of $L_{1}=L$. Numerical analytical results show that the following conclusions can be obtained by ANSYS software. (1) The temperature distributions of point $\mathrm{D}$ and point $\mathrm{E}$ are basically the same. The errors of point $\mathrm{C}$ temperature and point $\mathrm{E}$ temperature are very small, about $12.5^{\circ} \mathrm{C}$, therefore, the temperature distributions of actual tip point $\mathrm{D}$ and equivalent tip point $\mathrm{C}$ are considered to be the same in the whole period of time. (2) For point $\mathrm{D}$, the moment producing the maximum axial heat stress is the moment of maximum temperature gradient between point $\mathrm{B}$ and point D. (3) For the part of bar having oblique line, the temperature distribution of every point with the same radius is the same. (4) The stress concentration effect of V-shaped notch has a great influence on the heat crack of $\mathrm{V}$-shaped notch tip.

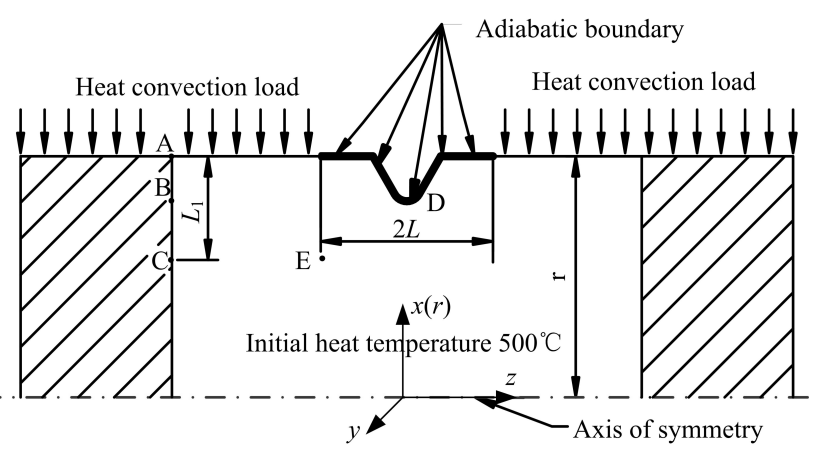

Fig. 3. The mathematical model of the metal bar

In view of the cooling water affecting the circumferential surface of metal bar at any time, its temperature field is an axisymmetric temperature field. By means of the mathematical model of the metal bar in Fig.3, the heat conduction differential equation in cylindrical coordinates is expressed by

$$
\frac{\partial t}{\partial \tau}=\alpha\left(\frac{\partial^{2} t}{\partial r^{2}}+\frac{1}{r} \frac{\partial t}{\partial r}\right)
$$

Where $\alpha$ is the coefficient of temperature conductivity, $t$ is the temperature of bar interior point, $\tau$ is the cooling time and $r$ is the polar coordinate.

When the bar has a convection heat exchange with the cooling water, the temperature of bar interior point is affected by the bar surface load. At the same time, the change of bar internal temperature also affects the convection heat transfer of the bar surface. Therefore, in order to simplify the calculation, the convective heat transfer process and the internal heat conduction are strictly separated in this paper, namely, the convective heat transfer process of the bar surface is regarded as the boundary 
condition of heat conduction, which is very important to calculate the heat conduction process rapidly and accurately. Based on it, the theoretical expression of unsteady multidimensional temperature field for the axisymmetric model of metal bar is obtained by

$$
T=\frac{2 T_{10} h_{1}^{2}}{b} \sum_{n=1}^{\infty} \frac{J_{0}\left(p_{n} r\right)}{\left(h_{1}^{2}+p_{n}^{2}\right) p_{n} J_{1}\left(p_{n} b\right)} e^{-p_{n}^{2} \alpha \tau}+T_{1}
$$

Where $T_{10}$ is the difference of initial temperature of the bar and the cooling water temperature, $T_{1}$ is the temperature of cooling water, $b$ is the radius of the bar, $J_{v}(x)$ is the bessel function, $p_{n}$ is the positive root of $p J_{1}(p b)-h_{1} J_{0}(p b)=0$, and $h_{1}$ is the relative coefficient of convective heat transfer, it can be calculated by

$$
h_{1}=C\left(\frac{u 2 b}{v_{f}}\right)^{n} k_{f} \operatorname{Pr}_{m}^{1 / 3} /(2 b \lambda)
$$

Where $u$ is the water velocity, $v_{f}$ is the kinematic viscosity of cooling water, $k_{f}$ is the thermal conductivity of saturated water, $\operatorname{Pr}_{m}$ is the Prandtl number of saturated water, $\lambda$ is the thermal conductivity of bar materials, $C$ and $n$ are constant at different Reynolds numbers.

\subsection{Analysis of temperature field in the bar}

When the radius of the metal bar is $7.5 \mathrm{~mm}$, the depth of $\mathrm{V}$ shaped notch is $0.8 \mathrm{~mm}$, the temperature of cooling water is $25^{\circ} \mathrm{C}$, and the initial temperature of the metal bar is $500^{\circ} \mathrm{C}$, the curves of point $\mathrm{B}$ temperature and point $\mathrm{C}$ temperature and time are obtained in Fig. 4 and Fig.5 respectively under different water velocities. As shown in Fig. 4 and Fig.5, the temperature of bar interior point decreases with the increase of water velocity at the same time. The temperature of point $\mathrm{C}$ is higher than one of point $\mathrm{B}$ in the condition of the same water velocity at the same time. However, for point $C$ and point $\mathrm{B}$, the time for reaching the final equilibrium temperature is almost the same.

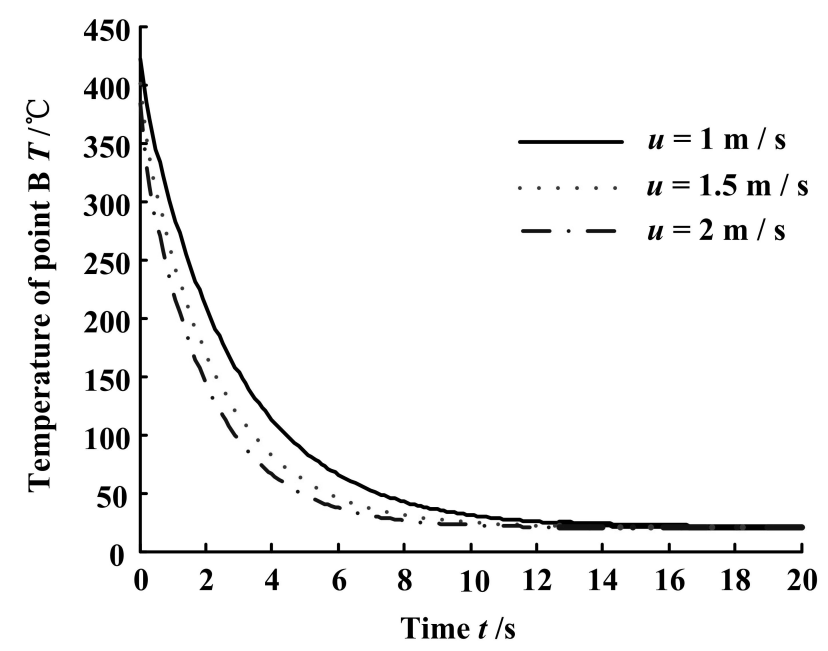

Fig. 4. The curve of point B temperature and time

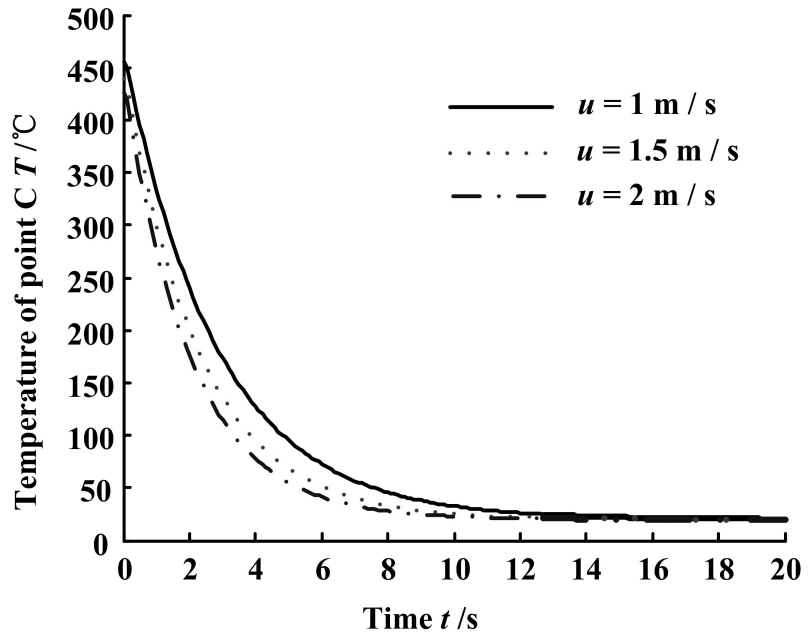

Fig. 5. The curve of point $\mathrm{C}$ temperature and time

\section{Critical temperature gradient of germinating heat crack in the tip of $\mathrm{V}$-shaped notch}

According to the elastic mechanics, the relation of the stress $\sigma$ and the strain $\varepsilon$ in the elastic range is expressed by

$\sigma=E \varepsilon$

Where $E$ is elastic modulus of material. The relation of elastic modulus of material and temperature for 45 steel is expressed by [12]

$E=2.1244 \times 10^{5} e^{-0.0003648 T}$

When the temperature difference of two different parts of the bar is $\Delta T$, the relation between the strain and temperature difference is expressed by

$\varepsilon=\Delta T a$

Where $a$ is the linear thermal expansion coefficient of material and it is equal to $11475 \times 10^{-9}+10.6 \times 10^{-9} \mathrm{~T}$.

When the heat stress of $\mathrm{V}$-shaped notch bottom, which is caused by the temperature gradient between point $\mathrm{C}$ and point $\mathrm{B}$, arrives at the fracture strength $\sigma_{f}$ of the material, the bottom of bar V-shaped notch is thought to produce the heat crack in this paper. The fracture strength $\sigma_{f}$ of the material is calculated by [13]

$$
\sigma_{f}=\sigma_{b}\left(1+\psi_{k}\right)
$$

Where $\psi_{k}$ is the section shrinkage ratio of material and $\sigma_{b}$ is the ultimate strength of material. The relation of the ultimate strength of material and the temperature for 45 steel is obtained by the polynomial curve fitting method.

$$
\begin{aligned}
\sigma_{b}= & 7.5631 \times 10^{-2} T^{4}-88.9622 T^{3}+30514.1258 T^{2} \\
& -3076191.99 T+688.9 \times 10^{6}
\end{aligned}
$$


The critical temperature gradient of germinating heat crack in the tip of $\mathrm{V}$-shaped notch is obtained by Eqs.(2)-(8)

$$
\Delta T=T_{c}-T_{b}=\frac{\sigma_{b}\left(1+\psi_{k}\right)}{E a}
$$

Supposing $T_{x}=T_{c}-\frac{\sigma_{b}\left(1+\psi_{k}\right)}{E \alpha}, T_{y}=T_{b}$. If curve $T_{x}$ and curve $T_{y}$ have a point of intersection, the critical temperature gradient of germinating the heat crack in the tip of V-shaped notch can be obtained. Linear thermal expansion coefficient, ultimate strength and elastic modulus are the function of temperature $T_{c}$ respectively in the calculation course.

\section{Influences of width of the adiabatic boundary and water velocity on the critical temperature gradient}

5.1 Influence of width of the adiabatic boundary on the critical temperature gradient

When the width of adiabatic boundary is $2 \mathrm{~mm}$, which is also equal to half of asbestos clapboard width in Fig.2, water velocity is $2 \mathrm{~m} / \mathrm{s}$, curve $T_{x}$ and curve $T_{y}$ have not a point of intersection as shown in Fig.6. When the width of adiabatic boundary is $3.745 \mathrm{~mm}$, water velocity is $2 \mathrm{~m} / \mathrm{s}$, curve $T_{x}$ and curve $T_{y}$ have a point of intersection in 0.2 seconds as shown in Fig.6. At present, $T_{c}$ is equal to $507.6^{\circ} \mathrm{C}$, and $T_{B}$ is equal to $331{ }^{\circ} \mathrm{C}$. Because the temperature of point $\mathrm{C}$ has exceeded the original temperature $500{ }^{\circ} \mathrm{C}$ of the bar, the width $3.745 \mathrm{~mm}$ of adiabatic boundary does not meet the actual situation. However, the analytical results also show that the heat crack can occur by improving the initial temperature of the metal bar.

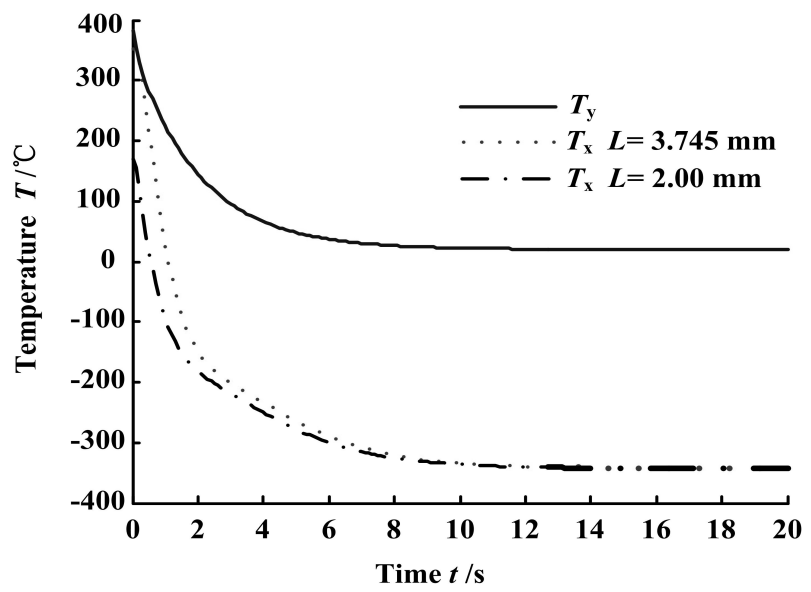

Fig. 6. Curve $T_{x}$ and curve $T_{y}$ in $\mathrm{L}=3.745 \mathrm{~mm}, \mathrm{~L}=2 \mathrm{~mm}$

To further analyze the influence of width of adiabatic boundary on the critical temperature gradient in the bar, the analytical results are also obtained in Fig.7 when the width of adiabatic boundary is $5.0 \mathrm{~mm}$ and $4.0 \mathrm{~mm}$ respectively. As is shown in Fig.7, when the width of adiabatic boundary is $5 \mathrm{~mm}$, water velocity is $2 \mathrm{~m} / \mathrm{s}$, curve $T_{x}$ and curve $T_{y}$ have a point of intersection in 0.628 seconds. At present, $T_{c}$ is equal to $472.36^{\circ} \mathrm{C}$, and $T_{B}$ is equal to $266.31{ }^{\circ} \mathrm{C}$. When the width of adiabatic boundary is $4 \mathrm{~mm}$, water velocity is $2 \mathrm{~m} / \mathrm{s}$, curve $T_{x}$ and curve $T_{y}$ have a point of intersection in 0.35 seconds. At present, $T_{c}$ is equal to $492.9^{\circ} \mathrm{C}$, and $T_{B}$ is equal to $304.55^{\circ} \mathrm{C}$. In a word, the critical temperature gradient can be carried out by changing the width of adiabatic boundary under the condition of the same water velocity. At the same time, the corresponding temperature gradient increases with the increase of width of adiabatic boundary.

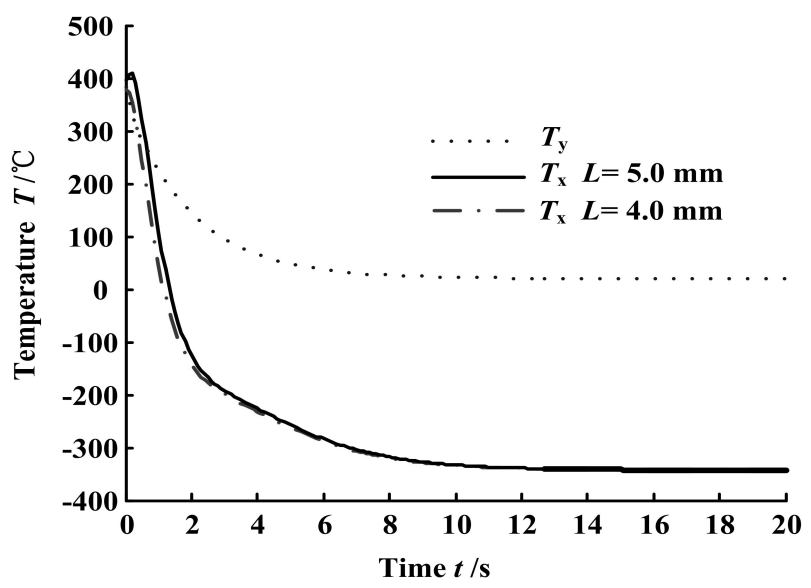

Fig. 7. Curve $T_{x}$ and curve $T_{y}$ in $\mathrm{L}=4 \mathrm{~mm}, \mathrm{~L}=5 \mathrm{~mm}$

5.2 Influence of water velocity on the critical temperature gradient

When the width of adiabatic boundary is $4 \mathrm{~mm}$, the influence of water velocity on the critical temperature gradient between point $\mathrm{C}$ and point $\mathrm{B}$ is shown in Fig. 8. As is shown in Fig.8, all of the critical temperature gradients can be carried out under the condition of water velocity $1 \mathrm{~m} / \mathrm{s}, 2 \mathrm{~m} / \mathrm{s}$, $3.5 \mathrm{~m} / \mathrm{s}$. However, the corresponding temperature gradient and the water spraying time are different under the different water velocities. When the water velocity is $1 \mathrm{~m} / \mathrm{s}$, the corresponding temperature gradient is $195.89^{\circ} \mathrm{C}$ and the water spraying time is about $0.467 \mathrm{~s}$. When the water velocity is $2 \mathrm{~m} / \mathrm{s}$, the corresponding temperature gradient is $188.35^{\circ} \mathrm{C}$ and the water spraying time is about $0.35 \mathrm{~s}$. When the water velocity is $3.5 \mathrm{~m} / \mathrm{s}$, the corresponding temperature gradient is $182.05^{\circ} \mathrm{C}$ and the water spraying time is about $0.258 \mathrm{~s}$. In a word, the water spraying time and the corresponding temperature gradient increase with the decrease of water velocity under the condition of the same width of adiabatic boundary.

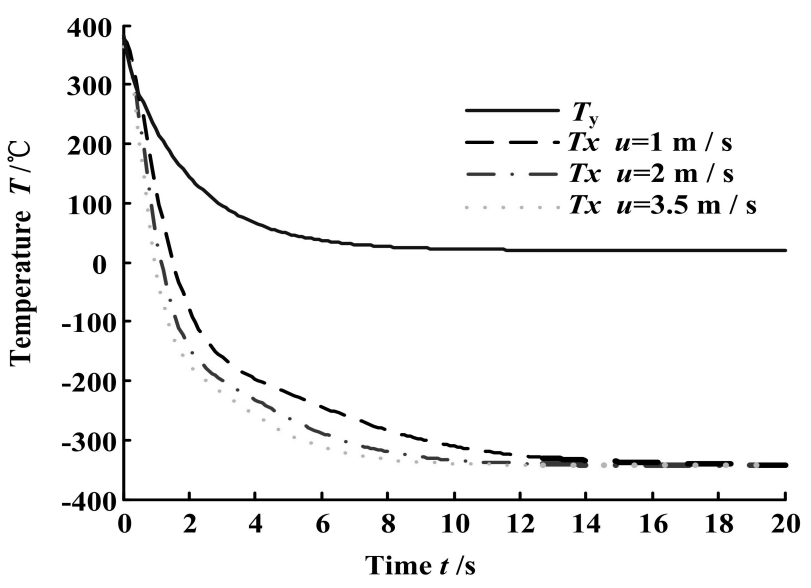

Fig. 8. Influence of water velocity on the critical temperature gradient 


\section{Experimental results}

When the radius of the metal bar is $7.5 \mathrm{~mm}$, the depth of $\mathrm{V}$ shaped notch is $0.8 \mathrm{~mm}$, the temperature of cooling water is $25^{\circ} \mathrm{C}$, and the initial temperature of the bar is $500^{\circ} \mathrm{C}$, by mean of heat stress experiment and the cropping experiment, the experimental results of water spraying time of having the heat crack in different water velocities and different widths of adiabatic boundary are shown in Table 1 . As is shown in Table 1, the experimental results of water spraying time are consistent with the above theoretical analysis results. The errors between the experimental results and the above theoretical analysis results are within $5 \%$. It further proves that the theoretical expression of unsteady multidimensional temperature field for the axisymmetric model proposed in the paper is feasible in the rapid calculation of crack generation. The experimental results also show that the geometric parameters of $\mathrm{V}$-shaped notch, the temperature of the cooling water, and the initial heat temperature of the metal bar have important influences on the water spraying time and critical temperature gradient between point $\mathrm{C}$ and point B to some extent.

Table 1: Water spraying time (s) of having the heat crack

\begin{tabular}{ccccc}
\hline $\begin{array}{c}\text { Water } \\
\text { velocity } \\
(\mathrm{m} / \mathrm{s})\end{array}$ & 2 & 3.745 & 4 & 5 \\
\hline 1 & $\times$ & 0.32 & 0.48 & 0.75 \\
\hline 2 & $\times$ & 0.23 & 0.36 & 0.64 \\
\hline 3.5 & 0.19 & 0.21 & 0.26 & 0.49 \\
\hline
\end{tabular}

\section{Conclusions}

(1) The theoretical expression of unsteady multidimensional temperature field for the axisymmetric model of metal bar is obtained in detail in this paper. The experimental results show that the expression is feasible in the rapid calculation of crack generation of $\mathrm{V}$-shaped notch tip.

(2) The critical temperature gradient can be carried out by changing the width of adiabatic boundary under the condition of the same water velocity. At the same time, the corresponding temperature gradient increases with the increase of width of adiabatic boundary. Theoretical analysis results also show that there should be an optimal water velocity and width of adiabatic boundary, which can minimize the critical temperature gradient.

(3) The water spraying time and the corresponding temperature gradient increases with the decrease of water velocity under the condition of the same width of adiabatic boundary.

\section{Acknowledgements}

The authors wish to express gratitude to the National Natural Science Foundation of PR China (Approval no. 51105382), Shandong Natural Science Foundation of PR China (Approval no. ZR2011EL039) and Qingdao Development Zone Science and Technology Development Project (Approval no. 2011-2-50), which have supported this work.

\section{References}

1. J.D. Chen, Y.W. Wang, D.H. Yu, et al, Brittle precision cropping of metal materials. Int. J. Mach. Tools Manuf, 32(3), 1992, pp 415424

2. L.C. Chan, T.C. Lee, B.J. Wu, et al, Experimental study on the shearing behavior of fine-blanking versus bar cropping. J. Mater. Process. Technol, 80-81, 1998, pp 126-130

3. Lijun Zhang, Yongrui, Zhao, Hengfu Xiang, Research of Stress Intensity Factor of V-shaped Notch Tip in Precision Cropping. International Journal of Advanced Manufacturing Technology, 65(1), 2003, pp 549-555

4. Lijun Zhang, Hengfu Xiang, Shijing Wang, Research on the cropping technology key parameters with variable-speed circumferential low-stress bend fracture. Machine Tool \& Hydraulics, 39(7), 2011, pp 69-70,123

5. Y. Tang, S.D. Zhao, J. Lin, Experimental investigation of the effect of the circumferential loading of a rotary striking action cropping system. Proc. IMechE, Part B: Journal of Engineering Manufacture, 224(7), 2010, pp 1095-1101

6. Y. Tang, S.D. Zhao, W.Z. Wang, A novel type of precision cropping machinery using rotary striking action. Proc. IMechE, Part C: Journal of Mechanical Engineering Science, 223(9), 2009, pp 1965-1967
7. Shengdun Zhao, Lijun Zhang, Wei Liu, Chunjian Hua, Feasibility investigation of ideal fracture caused by thermal stress. Journal of Plasticity Engineering, 13(5), 2006, pp 51-57

8. S.D. Zhao, L.J. Zhang, J. Lei, et al, Numerical study on heat stress prefabricating ideal crack at the bottom of $\mathrm{V}$ shaped notch in precision cropping. J. Mater. Process. Technol, 187-188, 2007, pp 363-367

9. L.J. Zhang, S.D. Zhao, Influence of cooling water temperature and initial heat temperature of bar on heat stress at the bottom of Vshaped notch. AIP Conference Proceedings, 1060(1), 2008, pp 208-211

10. Lijun Zhang, Yongrui, Zhao, Shengdun Zhao, Improvement of experimental device for prefabricating $\mathrm{V}$ - shaped notch tip crack. Manufacturing Technology \& Machine Tool, (10), 2012, pp 128130

11. H. Min, Y. Yue, W. Ling, Mechanics of materials. Xi'an Jiaotong University Press, Xi'an, 1999

12. Z.D. Yan, H.L. Wang, Heat stress. Higher Education Press, Beijing, 1993

13. S.M. Yang. Heat transfer theory. Higher Education Press, Beijing, 1991 